\title{
IDENTIFICAÇÃO MORFOLÓGICA DE FLEBOTOMÍNEOS CAPTURADOS EM ÁREA URBANA
}

\author{
MORPHOLOGIC IDENTIFICATION OF PHLEBOTOMINE SANDFLIES \\ CAPTURED IN URBAN AREA
}

\author{
Thiago Souza Azeredo Bastos ${ }^{1 *}$ \\ Guido Fontgalland Coelho Linhares ${ }^{1}$ \\ Darling Melany de Carvalho Madrid ${ }^{1}$ \\ ${ }^{1}$ Escola de Veterinária e Zootecnia da Universidade Federal de Goiás, Goiânia, GO \\ *Autor para contato - tsabvet@gmail.com
}

\section{Resumo}

Flebotomíneos tem considerável importância na transmissão de agentes etiológicos de doenças tais como bartonelose, arboviroses e especialmente a leishmaniose visceral e leishmaniose tegumentar americana, enfermidades presentes na cidade de Goiás. Com o objetivo de se identificar a população de flebotomíneos nesta localidade, foram realizadas capturas desses insetos na área urbana da cidade de Goiás. As armadilhas CDC foram posicionadas ao longo de toda área urbana, entre setembro de 2012 e agosto de 2013, no último final de semana de cada mês, repetidamente por três noites seguidas. Além da captura de insetos, foram coletados dados de umidade, precipitação e temperatura. Os resultados obtidos foram analisados por meio de estatística descritiva e analisados pela correlação de Pearson ao nível de significância de 5\%. Foram coletados 342 flebotomíneos, pertencentes a oito espécies: Lutzomyia longipalpis, Nyssomyia whitmani, Nyssomyia intermedia, Evandromyia lenti, Psathyromyia shannoni, Micropygomyia peresi, Evandromyia bacula e Micropygomyia goiana. Este é o primeiro registro de Ev. bacula, Mi. peresi e Mi. goiana para a cidade de Goiás.

Palavras-chave: Ev. bacula, Goiás, leishmanioses, Mi. goiana, vetores.

\begin{abstract}
Phlebotomine sandflies have considerable importance in the transmission of the etiological agents of diseases, such as bartonellosis, arboviruses, and especially visceral and cutaneous leishmaniasis, diseases present in the city of Goiás. In order to know the population of sand flies in this locality, collections of these insects have been taken in the urban area of the city of Goiás. CDC traps were placed throughout the urban area between September 2012 and August 2013, in the last weekend of each month, for three consecutive nights. In addition to capturing insects, we collected humidity, precipitation and temperature data. The results obtained through Pearson test showed a weak relationship between the amount of gathered sand flies with humidity. A total of 342 sand flies were collected, belonging to eight species: Lutzomyia longipalpis, Nyssomyia whitmani, Nyssomyia intermedia, Evandromyia lenti, Psathyromyia shannoni, Micropygomyia peresi, Evandromyia bacula and Micropygomyia goiana. This is the first record of Ev. bacula, Mi. peresi and Mi. goiana to the city of Goiás.
\end{abstract}

Keywords: Ev. bacula, Goiás, leishmaniasis, Mi. goiana, vectors.

Enviado em: 08 outubro de 2014

Aceito em: 08 junho de 2016 


\section{Introdução}

Flebotomíneos são dípteros com considerável importância na sanidade animal e na saúde pública, pois estão envolvidos na transmissão de protozoários do gênero Leishmania, que causam a leishmaniose visceral (LV) e a leishmaniose tegumentar americana (LTA) em animais e humanos, no meio urbano e rural em diferentes estados do Brasil ${ }^{(1)}$.

No Brasil, a LV é causada por Leishmania (Leishmania) infantum chagasi e é transmitida por flebotomíneos das espécies Lutzomyia longipalpis e Lutzomyia cruzi $^{(2)}$. A LTA é causada pelas espécies L. (Viannia) braziliensis, L. (Viannia) guyanensis, L. (Viannia) lainsoni, L. (Viannia) naiffi, L. (Viannia) shawi, L. (Viannia) lindenbergi e L. (Leishmania) amazonenses ${ }^{(3)}$ e os vetores mais conhecidos são os flebotomíneos Bichromomyia flaviscutellata, Nyssomyia intermedia, Migonemyia migonei e Nyssomyia whitmani ${ }^{(4)}$.

Entre 1999 e 2002 foram registrados dez casos humanos de leishmanioses no município de Goiás, Estado de Goiás, dos quais três de LTA e sete de LV. Nessa mesma ocasião, a pesquisa de flebotomíneos nas residências dos indivíduos acometidos revelou a presença das espécies Lu. longipalpis, $N y$. whitmani, Ny. intermedia, Evandromyia lenti, Evandromyia termitophila, Psathyromyia shannoni, Psychodopygus lainsoni, Pintomyia pessoai ${ }^{(5)}$.

A persistência de casos humanos de LV e $\mathrm{LTA}^{(6)}$ e a inexistência de um trabalho continuado sobre o vetor despertou a necessidade de se reavaliar a fauna de flebotomíneos, no município de Goiás, o que constituiu o objetivo desta investigação.

\section{Material e Métodos}

A pesquisa foi realizada durante o período de setembro de 2012 a agosto de 2013, na área urbana do município de Goiás, no noroeste do Estado de Goiás, Brasil, a $135 \mathrm{~km}$ da capital do Estado. Com 23.806 habitantes, tem altitude média de 512 metros acima do nível do mar, relevo bastante acidentado, clima tropical úmido com duas estações bem definidas (uma quente e chuvosa e outra fria e seca). A vegetação predominante é o cerrado e conta com boa disponibilidade de recursos hídricos ${ }^{(7)}$. O município de Goiás possui mais de 280 anos e é uma cidade turística devido ao seu reconhecimento como Patrimônio Cultural da Humanidade pela UNESCO, em 2001.

Seguindo indicações da Secretaria Municipal de Saúde, para a realização das coletas foram selecionados 10 pontos onde anteriormente foram detectados casos humanos de LV e/ou LTA (Figura 1).

Para a captura de flebotomíneos, foram utilizadas armadilhas luminosas do tipo CDC, conforme indicação do Ministério da Saúde ${ }^{(8)}$. As armadilhas foram instaladas entre às 16 horas e 19 horas de cada dia e retiradas entre 7 e 9 horas do dia seguinte, no peridomicílio, a um metro e meio de altura do solo e distância máxima de dois metros das prováveis fontes de alimentação dos flebotomíneos. Assim, as armadilhas 1 (15 $\left.5^{\circ} 56^{\prime} 25 \mathrm{~S} ; 50^{\circ} 09^{\prime} 32^{\circ} \mathrm{W}\right), 2\left(15^{\circ} 56^{\prime} 49 \mathrm{~S} ; 50^{\circ} 09^{\prime} 11^{\circ} \mathrm{W}\right)$, $3 \quad\left(15^{\circ} 56^{\prime} 92 \mathrm{~S} ; 50^{\circ} 08^{\prime} 86^{\circ} \mathrm{W}\right), \quad 4 \quad\left(15^{\circ} 58^{\prime} 01 \mathrm{~S} ; 50^{\circ} 07^{\prime} 32^{\circ} \mathrm{W}\right), \quad 5 \quad\left(15^{\circ} 57^{\prime} 58 \mathrm{~S} ; 50^{\circ} 07^{\prime} 49^{\circ} \mathrm{W}\right), \quad 9$ $\left(15^{\circ} 55^{\prime} 89 \mathrm{~S} ; 50^{\circ} 08^{\prime} 41^{\circ} \mathrm{W}\right)$ e $10\left(15^{\circ} 56^{\prime} 52 \mathrm{~S} ; 50^{\circ} 08^{\prime} 40^{\circ} \mathrm{W}\right)$ foram instaladas próximas a galinheiros. As armadilhas $6\left(15^{\circ} 57^{\prime} 55 \mathrm{~S} ; 50^{\circ} 07^{\prime} 41^{\circ} \mathrm{W}\right)$ e $7\left(15^{\circ} 56^{\prime} 38 \mathrm{~S} ; 50^{\circ} 08^{\prime} 27^{\circ} \mathrm{W}\right)$ foram instaladas em canil e a armadilha $8\left(15^{\circ} 56^{\prime} 196 \mathrm{~S} ; 50^{\circ} 08^{\prime} 739^{\circ} \mathrm{W}\right)$ foi instalada em um curral. As coletas foram realizadas mensalmente, durante três noites consecutivas, perfazendo um total de 36 noites e 360 capturas. 


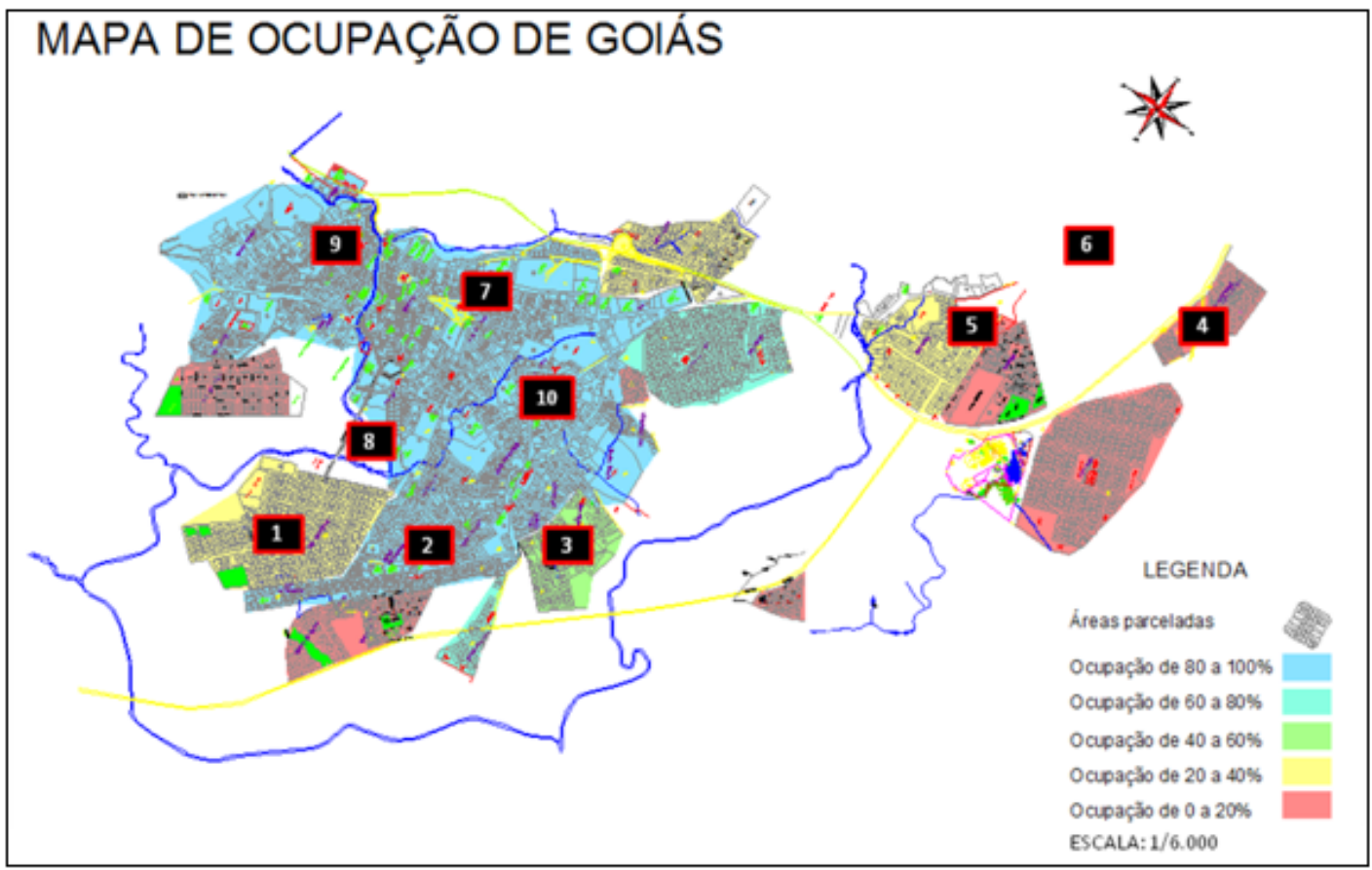

Figura 1: Distribuição das armadilhas na zona urbana do município de Goiás, estado de Goiás.

Os espécimes capturados foram sacrificados em vapores de éter ${ }^{(9)}$. Em seguida, os insetos foram armazenados em frascos plásticos contendo álcool $70 \%$ e identificados com data e local de captura ${ }^{(10)}$. Todos os frascos contendo os insetos coletados foram remetidos ao Laboratório de Doenças Parasitárias do Setor de Medicina Veterinária Preventiva, da Escola de Veterinária e Zootecnia (EVZ) da Universidade Federal de Goiás (UFG), onde foram triados em microscópio estereoscópico para a separação dos flebotomíneos. Em seguida os flebotomíneos foram preparados para identificação, conforme Galati ${ }^{(11)}$. Após a secagem das lâminas, os flebotomíneos foram identificados seguindo-se a chave de Young e Duncan ${ }^{(12)}$ e arquivados no referido laboratório.

Dados de temperatura (máxima, mínima e média), umidade e precipitação foram obtidos junto ao Instituto Nacional de Meteorologia ${ }^{(13)}$. Os valores representativos de cada coleta mensal resultam da média calculada para os três dias de captura.

Os resultados foram apresentados usando estatística descritiva e analisados pela correlação de Pearson a 5\% de significância, processada por meio do software livre " $R$ " (Lucent technologies $®$ ). Todas as análises estatísticas foram avaliadas com nível de significância $\alpha \leq 0,05$.

\section{Resultados e Discussão}

Foram capturados 342 flebotomíneos distribuídos em oito espécies (Tabela 1). As espécies identificadas foram Lu. longipalpis, Ny. whitmani, Ny. intermedia, Ev. lenti, Pa. shannoni, Mi. peresi, Ev. bacula e Mi. goiana. Notou-se predomínio das espécies Nyssomyia whitmani, correspondendo a $71,6 \%$, Lutzomyia longipalpis $(17,5 \%)$, Evandromyia lenti $(7,3 \%)$ e Nyssomyia intermedia $(1,8 \%)$. As demais espécies representam menos de $1 \%$ cada.

Deste total, 264 (77\%) eram machos e 78 (23\%) fêmeas. Ambos os sexos das espécies Lu. longipalpis, Ny. whitmani, Ev. lenti e Ny. intermedia foram identificados. Não foram capturados insetos machos das espécies Mi. peresi, Pa. shannoni, Lu. goiana e Ev. bacula. 
Tabela 1: Flebotomíneos coletados no município de Goiás, Estado de Goiás, de setembro de 2012 a agosto de 2013

\begin{tabular}{lcccc}
\hline \multirow{2}{*}{ Espécie } & \multicolumn{2}{c}{ Sexo } & & \\
\cline { 2 - 3 } & Macho & Fêmea & Total & $\%$ \\
\hline Nyssomyia whitmani & 200 & 45 & 245 & 71,6 \\
Lutzomyia longipalpis & 50 & 10 & 60 & 17,5 \\
Evandromyia lenti & 13 & 12 & 25 & 7,3 \\
Nyssomyia intermedia & 1 & 5 & 6 & 1,8 \\
Micropygomyia goiana & 0 & 3 & 3 & 0,9 \\
Micropygomyia peresi & 0 & 1 & 1 & 0,3 \\
Evandromyia bacula & 0 & 1 & 1 & 0,3 \\
Psathyromyia shannoni & 0 & 1 & 1 & 0,3 \\
\hline Total & 264 & 78 & 342 & 100,00 \\
\hline
\end{tabular}

A razão sexual (Macho/Fêmea) foi de 4,44/1 para Ny. whitmani, 5,00/1 para Lu. longipalpis e 1,08/1 para $E v$ v. lenti. Esses resultados diferem daqueles publicados por Rodrigues et al. ${ }^{(14)}$, em que a diferença na proporção entre macho e fêmea não foi significativa, e também diferem de Peréz et al. ${ }^{(10)}$, pois estes observaram que as armadilhas CDC capturaram preferencialmente fêmeas em seus estudos. Desta forma, ainda permanece incerto a relação entre as armadilhas tipo CDC e o sexo dos flebotomíneos capturados.

Levando em consideração o total de insetos capturados por ano, este quantitativo foi muito inferior aos resultados obtidos em outras cidades como: Dracena (média de 6.872 por ano) no estado de São Paulo, Barra do Garças (média de 4.048 por ano) no estado de Mato Grosso e Campo Grande (1.245 em um ano) no Mato Grosso do $\mathrm{Sul}^{(2,3,15)}$.

Com relação à diversidade, Martins et al. ${ }^{(5)}$ identificaram o mesmo número de espécies em Goiás em 2002, das quais seis foram novamente encontradas. As espécies Ev. termitophila, Ps. lainsoni e $P i$. pessoa não foram capturadas neste estudo. Por outro lado, Mi. peresi, Ev. bacula e Mi. goiana foram incorporadas à lista da fauna de flebotomíneos do município de Goiás, agora contando com onze espécies registradas. Mais uma vez, representa um quantitativo bem inferior quando comparado ao número de espécies já conhecidas para outros municípios já estudados (27 espécies em Barra do Garças $^{(2)}$ e 29 em Campo Grande), mas a diversidade de espécies foi superior quando comparado à Dracena, onde apenas cinco espécies foram detectadas ${ }^{(15)}$.

Avaliando-se os resultados de acordo com os diferentes ecótopos (Tabela 2), observou-se que 90\% dos flebotomíneos (310 insetos) foram capturados em canil. Uma pequena parte, 9\% (28 insetos), foram capturados em galinheiro e apenas 1\% (4 insetos) em curral. As espécies Lu. longipalpis e Ev. lenti foram encontradas nos três ecótopos. $N y$. whitmani e Mi. goiana foram encontradas em canil e galinheiro, $\mathrm{Ny}$. intermedia e Mi. peresi foram capturadas apenas em canil, $\mathrm{Pa}$. shannoni apenas em galinheiro e Ev. bacula apenas em curral.

Os dados meteorológicos, juntamente com o número de flebotomíneos capturados, estão apresentados nas Figuras 2 e 3. Verificou-se que a umidade ao longo dos doze meses variou de $24 \%$ a $80 \%$ (média de $55,41 \%$ ), a variação da temperatura máxima foi de 28 a $38^{\circ} \mathrm{C}$ (média de $32,58{ }^{\circ} \mathrm{C}$ ), a variação de temperatura mínima foi de 16 a $24{ }^{\circ} \mathrm{C}$ (média de $21,16{ }^{\circ} \mathrm{C}$ ), a temperatura média variou de 24 a $31{ }^{\circ} \mathrm{C}$ (média de $26,87^{\circ} \mathrm{C}$ ) e a precipitação variou de 0 a $409 \mathrm{~mm}$ (média de 104,08 $\mathrm{mm}$ e mediana igual a $71,50 \mathrm{~mm}$ ). Apenas a mediana da precipitaçãofoi apresentada, pois foi o único fator que apresentou valores discrepantes com a média.

Aplicando-se o coeficiente de correlação de Pearson entre o quantitativo de flebotomíneos capturados e os fatores meteorológicos, observou-se fraca correlação positiva $(0,07)$ com a umidade. Para os demais fatores, a correlação ou foi negativa (temperatura máxima foi de $-0,40$ e temperatura média de -0,33) ou tendeu à nulidade (temperatura mínima de -0,09 e precipitação de -0,05). 
Tabela 2: Flebotomíneos mais frequentes segundo a espécie e o ecótopo de captura no município de Goiás, Estado de Goiás, de setembro de 2012 a agosto de 2013

\begin{tabular}{ccccccccc}
\hline & \multicolumn{7}{c}{ Espécies } \\
\cline { 2 - 8 } Ecótopo & $\begin{array}{c}\text { Nyssomyia } \\
\text { whitmani }\end{array}$ & $\begin{array}{c}\text { Lutzomyia } \\
\text { longípalpis }\end{array}$ & $\begin{array}{c}\text { Evandromyia } \\
\text { lenti }\end{array}$ & $\begin{array}{c}\text { Nyssomyia } \\
\text { intermedia }\end{array}$ & $\begin{array}{c}\text { Lutzomyia } \\
\text { goiana }\end{array}$ & $\begin{array}{c}\text { Micropygomyia } \\
\text { peresi }\end{array}$ & $\begin{array}{c}\text { Evandromyia } \\
\text { bacula }\end{array}$ & $\begin{array}{c}\text { Psathyromyia } \\
\text { shannoni }\end{array}$ \\
\hline Canil & $235(96 \%)$ & $55(92 \%)$ & $12(48 \%)$ & 6 & $1(34 \%)$ & 1 & 0 & 0 \\
Galinheiro & $10(4 \%)$ & $4(6 \%)$ & $11(44 \%)$ & 0 & $2(66 \%)$ & 0 & 0 & 1 \\
Curral & 0 & $1(2 \%)$ & $2(8 \%)$ & 0 & 0 & 0 & 1 & 0 \\
\hline Total & 245 & 60 & 25 & 6 & 3 & 1 & 1 & 1 \\
\hline
\end{tabular}

Entretanto, mesmo com a fraca correlação obtida, pôde-se observar que a menor taxa de captura de flebotomíneos ocorreu no primeiro semestre de trabalho (setembro a fevereiro), período que coincide com o início e o final do pico de chuvas no município. Por outro lado, o pico de captura ocorreu em abril (82,7\% da captura anual) e um comportamento crescente foi observado de maio a julho, justamente quando a precipitação reduz e a umidade atinge valores entre 40 e $60 \%$. Estes dados coincidem com os de outros autores, que apontaram o número maior de flebotomíneos capturados após o período de $\operatorname{chuvas}^{(15,16)}$.

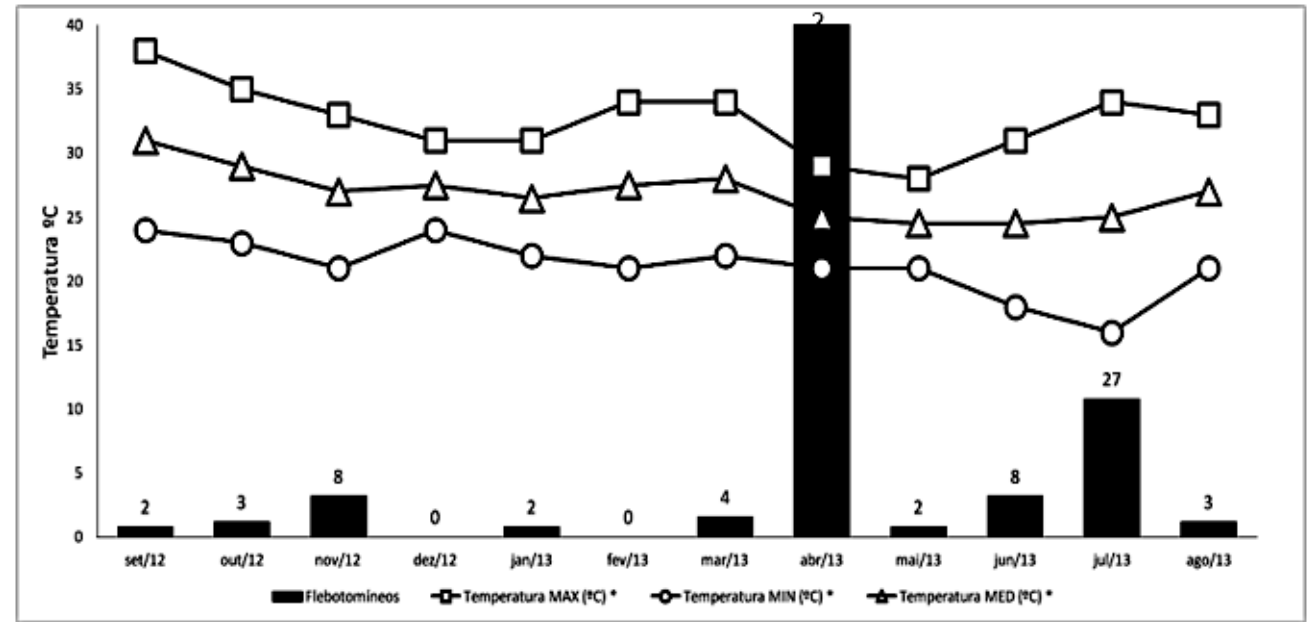

Figura 2: Variação sazonal do número de flebotomíneos e temperatura (máxima, mínima e média) no município de Goiás, Estado de Goiás, entre setembro de 2012 e agosto de 2013.

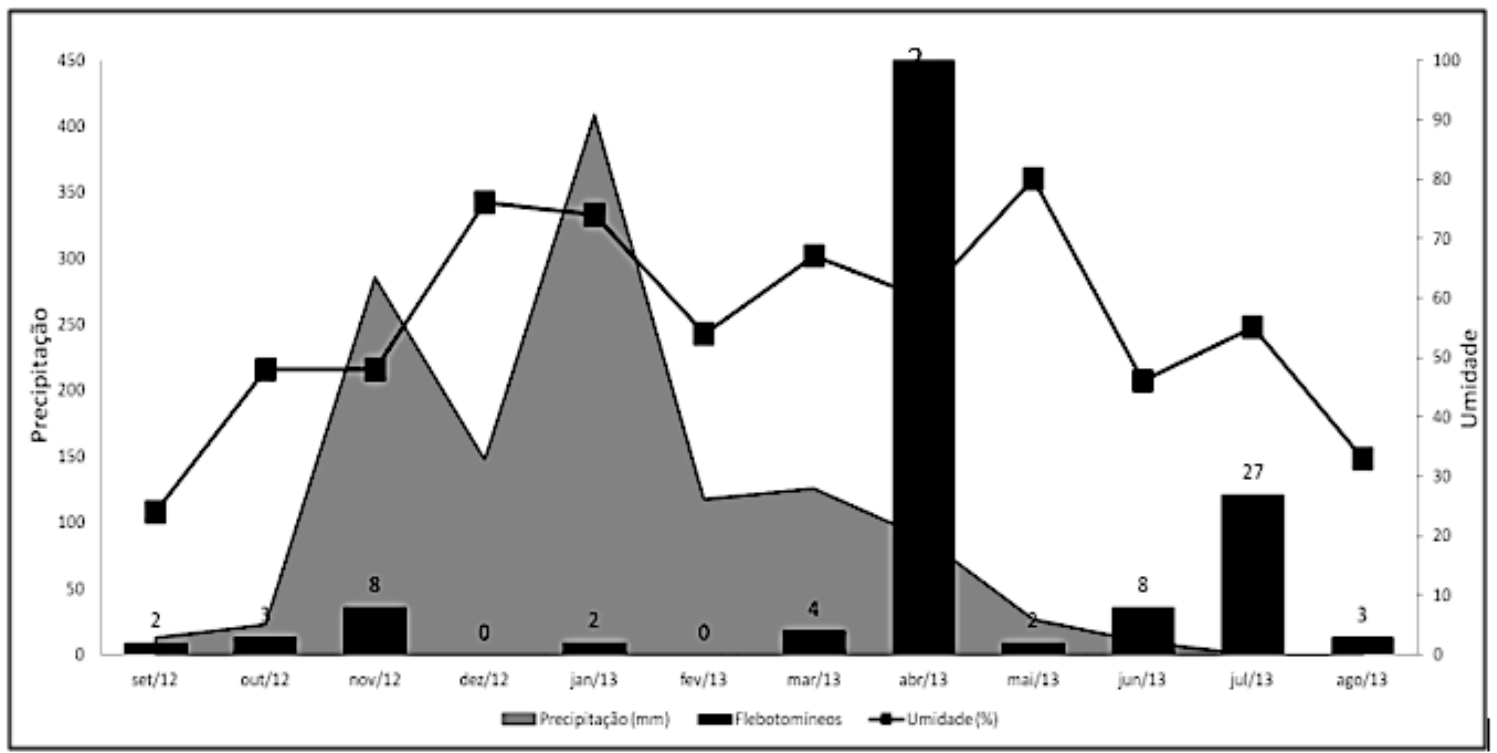

Figura 3: Variação sazonal do número de flebotomíneos, precipitação e umidade no município de Goiás, Estado de Goiás, entre setembro de 2012 e agosto de 2013. 


\section{Conclusões}

Durante o período de setembro de 2012 a agosto e 2013, flebotomíneos foram capturados em todos os pontos amostrados distribuídos ao longo de toda a cidade de Goiás. Foram capturadas e identificadas as seguintes espécies de flebotomíneos: Lu. longipalpis, $N y$. whitmani, $N y$. intermedia, Ev. lenti, Pa. shannoni, Ev. bacula, Mi. peresi e Lu. goiana. Este foi o primeiro registro de ocorrência das espécies Ev. bacula, Mi. peresi e Lu. goiana na cidade de Goiás.

Neste estudo, as armadilhas tipo CDC capturaram flebotomíneos machos numa proporção maior do que fêmeas. Contudo, essa correlação ainda precisa ser mais explorada.

Aparentemente, pode-se supor que houve relação entre a umidade relativa do ar e a precipitação com o número de flebotomíneos capturados. Entretanto, não foi observada correlação estatística entre os mesmos, assim como também não se observou correlação com os dados de temperatura (máxima e mínima).

\section{Referências}

1.Dantas-Torres F. Canine leishmaniosis in South America. Parasites \& Vectors. 2009; 2(1): 8p.

2.Queiroz MFM, Varjão JR, Moraes SC, Salcedo GE. Analysis of sandflies (Diptera: Psychodidae) in Barra do Garças, State of Mato Grosso, Brazil, and the influence of environmental variables on the vector density of Lutzomyia longipalpis (Lutz \& Neiva, 1912). Revista da Sociedade Brasileira de Medicina Tropical. 2012; 45(3): 313-17.

3. Oliveira AG, Andrade Filho JD, Falcão AL, Brazil RP. Estudo de flebotomíneos (Diptera, Psychodidae, Phlebotominae) na zona urbana da cidade de Campo Grande, Mato Grosso do Sul, Brasil, 1999-2000. Caderno de Saúde Pública. 2003; 19(4): 933-44.

4. Shimabukuro PHF, Galati EAB. Checklist dos Phlebotominae (Diptera, Psychodidae) do estado de São Paulo, Brasil, com comentários sobre sua distribuição geográfica. Biota Neotroprica. 2011; 11(1a).

5.Martins F, Silva IGC, Bezerra WA, Maciel J, Silva HHG, Lima CG, Cantuária PB, Ramos, OS, Bideiro JB, Santos AS. Diversidade e frequência da fauna flebotomínea (Diptera: Psychodidae) em áreas com transmissão de leishmaniose, no Estado de Goiás. Revista de Patologia Tropical. 2002; 31(2): 211-24.

6.SINAN. Sistema de Informação de Agravos de Notificação [online]. Brasília, Brasil; 2012. [capturado 12 fev.2012] Disponível em: www.saude.gov.br/sinan/. Prefeitura Municipal de Goiás. Secretaria Municipal de Turismo e Cultura, 2012.

7.Brasil. Ministério da Saúde. Manual de vigilância e controle da leishmaniose visceral. Editora do Ministério da Saúde, Brasília. 2006, p. 47, [Internet], disponível em http://bvsms.saude.gov.br/bvs/publicacoes/manual_ vigilancia_controle leishmaniose visceral.pdf, acesso em junho 2016.

8.Vilela ML, Rangel EF, Lainson R. Métodos de coleta e preservação de flebotomíneos, p.353-367. In Rangel EF \& Lainson R. Flebotomíneos do Brasil. Rio de Janeiro: Editora Fiocruz, 2003. 367 p.

9.Perez J, Virgen A, Rojas JC, Rebollar-Tellez EAA, Castillo IF, Mikery O, Marina CF, Ibanez-Bernal S. Species composition and seasonal abundance of sandflies (Diptera: Psychodidae: Phlebotominae) in coffee agroecosystems. Memórias do Instituto Oswaldo Cruz. 2013; 109(1): 80-86.

10.Galati EAB. Morfologia, terminologia de adultos e identificação dos táxons da América, p.53-176. In Rangel EF \& Lainson R. Flebotomíneos do Brasil. Rio de Janeiro: Editora Fiocruz, 2003. 367 p 
11.Young DG \& Duncan MA. Guide to the identification and geographic distribution of lutzomyia sand flies in Mexico, the West Indies, Central and South America (Diptera: Psychodidae). Gainesville: Memoirs of the American Entomological Institute. 1994; 54: 887 p.

12.INMET. Instituto Nacional de Meteorologia. Disponível em: http://www.inmet.gov.br/portal/index. php?r=home/page\&page=rede_estacoes_conv_graf, acesso em 08/02/2014.

13.Rodrigues AAF, Barbosa VA, Andrade Filho JD, Brazil RP. The sandfly fauna (Diptera: Psychodidae: Phlebotominae) of the Parque Estadual da Serra da Tiririca, Rio de Janeiro, Brazil. Memórias do Instituto Oswaldo Cruz. 2013; 108(7): 943-946.

14.Holcman MM, Sampaio SMP, Rangel O, Casanova C. Spatial and seasonal distribution of Lutzomyia longipalpis in Dracena, a city in the western region of the State of São Paulo, Brazil, that is endemic with visceral leishmaniasis. Revista da Sociedade Brasileira de Medicina Tropical. 2013; 46(6): 704-712.

15.Carreira-Alves JR. Espécies de Phlebotominae (Diptera: Psychodidade) da fazenda São José, município de Carmo, estado do Rio de Janeiro, Brasil. Revista de Patologia Tropical. 2009; 37(4): 371-372. 